\title{
Closing the Cognitive Gap between Humans and Interactive Narrative Agents Using Shared Mental Models
}

\author{
Rania Hodhod \\ Columbus State University \\ 4225 University Avenue, Columbus, GA \\ hodhod_rania@columbusstate.edu
}

\author{
Brian Magerko \\ Georgia Institute of Technology \\ North Ave NW, Atlanta, GA \\ magerko@gatech.edu
}

\begin{abstract}
This paper proposes a new formal approach for negotiating shared mental models between humans and computational improvisational agents (improv agents) based on our sociocognitive studies of human improvisers. Negotiation of shared mental models serves as a core mechanism for improv agents to co-create stories with each other and with human interactors. The model aims to narrow the gap between human and machine intelligence by providing AI agents that, in the presence of incomplete knowledge about an improv scene, can use procedural representations not only to understand human parties but also to negotiate their mental models with them. The described approach allows flexible modeling of ambiguous, non-Boolean knowledge through the use of fuzzy logic and situation calculus that allows reasoning under uncertainty in a dynamic improvisational setting.
\end{abstract}

\section{Author Keywords}

Computational creativity; cognitive consensus; fuzzy logic; improvisational agents; shared mental models.

\section{ACM Classification Keywords}

H.5.2 [User Interfaces]: Interaction Style; I.2.11

[Distributed Artificial Intelligence]: Intelligent Agents; I.2.3 [Deduction and Theorem proving]: Uncertainty, Fuzzy and belief revision.

\section{INTRODUCTION}

Interactive narrative research has heavily focused on systems that either provide a) a centralized drama manager agent that mediates a user's narrative experience through the behavior of weakly autonomous agents [1] or b) strongly autonomous systems that represent a story as the

Permission to make digital or hard copies of all or part of this work for personal or classroom use is granted without fee provided that copies are not made or distributed for profit or commercial advantage and that copies bear this notice and the full citation on the first page. Copyrights for components of this work owned by others than ACM must be honored. Abstracting with credit is permitted. To copy otherwise, or republish, to post on servers or to redistribute to lists, requires prior specific permission and/or a fee. Request permissions from Permissions@acm.org.

IUI'16, March 07-10, 2016, Sonoma, CA, USA

(C) 2016 ACM ISBN 978-1-4503-4137-0/16/03 ...\$15.00

DOI: http://dx.doi.org/10.1145/2856767.2856774 interaction of agents pursuing their goals [2-4]. Both of these approaches view interactive narrative systems as an embodiment of authorial intentions; story content is created by an author that is then exposed to the user, depending on the user's interactions/choices with the system. User contributions to the story do not actively create story elements in as much as move the experience in a particular direction within a pre-authored story space. This model puts the computer in a privileged position in the user/machine relationship. Whether story elements are represented as beats [5], plot points [6], [7], planning operators [2], or some other instantial content, contemporary interactive narrative systems privilege the computer over the human interactor as a creative entity in the narrative experience. These systems do not view interactive narrative as a cocreative experience-where each participant has an active role in the creation of story content- which is seen in such real life experiences as improvisational theatre or children playing pretend.

Improvisational theatre is a creative group performance where actors co-construct stories on stage in real time. There is typically no centralized story creator, but rather two or more improvisers who are perpetually negotiating elements of the scene in real time. First, improvisers attempt to define the platform of a scene as the introduction (e.g. scene location, characters, the activity they are engaged in, etc.) [8]. Then typically moves towards finding the tilt of the scene (i.e. the main conflict or problem to solve in the scene) [9]. Finally, a resolution to the tilt is then attempted, thus ending the scene.

Despite the fact that this general process of defining scene elements is all done as part of the performance on stage, the emergent narrative can still be represented as a plot, like any other play. However, improvisers do not go behind stage and agree on scene elements, nor does the moderator of the scene typically provide explicit scene elements to them ${ }^{1}$. Rather, the elements of a scene are

\footnotetext{
${ }^{1}$ The amount of detail given to performers can vary wildly from troupe to troupe. Some troupes practice giving the entire platform,
} 
perpetually negotiated as part of a performance (what Sawyer calls collaborative emergence [12]). Improvisers are continuously adding elements to the scene that have a degree of ambiguity to them (e.g. intending to communicate that one is a pirate by petting a parrot on their shoulder, which could also communicate that one is a zookeeper without any other context provided) or that may be rejected by the other improvisers for a variety of reasons (e.g. it conflicts with their view of the scene, disagrees with it being appropriate material for the audience, etc.). In other words, improvisers continuously negotiate a shared mental model (SMM) of what is true in the scene, such as the platform [13]. Our empirical studies of improvisers have shown that this process of building such a model, called collaborative convergence, is at the essence of improvised scenes [13].

Cognitive psychologists provide representations of the mental models that people form when understanding narratives and consequently how humans know, perceive, make decisions, and construct behavior in social situations [14]. A mental model typically contains minimal information (i.e. not every single detail but the main salient points of a phenomenon or experience) described as a set of well-defined, highly organized knowledge [15]. In other words, information is presented with the presumption that minimal processing effort is required [41]. Though, confidence about a particular piece of well-defined knowledge may vary. For example, "cowboys always wear hats" is a simple (Boolean) example of well-defined knowledge. However, people's confidence in this statement might vary from being completely confident $(100 \%)$ or completely unconfident $(0 \%)$, which indicates Boolean logic, or perhaps being partially confident in it. For example, being $60 \%$ confident about this statement and $40 \%$ uncertain is referred to as fuzzy logic. The confidence level in a specific piece of knowledge can act as an indication of the awareness of this knowledge [15].

The concept of mental models is extended to SMMs when it is related to team contexts [16]. SMMs are a hypothetical cognitive construct that incorporates the development of mutual beliefs from the team members' individual mental models until a common mental model is reached by the team members, either explicitly or implicitly. SMMs among team members develop from working and training together on similar tasks [17]. Studies by Yen et al. [17] suggest a close relationship between the existence of a shared mental model and successful team performance; the stronger the shared mental model the better tasks are accomplished. Shared mental models are essential in domains that require co-creation of stories on the fly like improvisation as they enable better

as seen in the popular television show, Whose Line Is It Anyway? [10], [11]. communication and mutual understanding between the actors.

The work presented in this paper provides new insights on co-creativity in interactive narrative through supplying the AI agents with the processes based in constructing shared mental models that can be used for supporting cocreative interactions with humans, the knowledge that those processes operate on, and the application of these processes in the improv theatre domain. Work on computational improvisation has yielded agents that model the cognitive processes central to the human act of theatrical improvisation, but relied on pre-authored narrative structures to construct a story with the user rather than providing the agents with capabilities that allow them to cocreate a new story with the user. The paper presents a computational shared mental model that allows improv agents to communicate with each other and with a human actor to reach mutual understanding about the identity of their characters and the nature of their joint activity.

The following section gives an overview of improvisational acting, defining the main cognitive skills involved in this domain and clarifying how human improvisers make use of mind reading [18] and theory of mind [19] to communicate with each other (we are interested in mindreading and theory of mind as they reflect one's ability to understand and interact with others by having some grasp of their mental states and using this understanding to explain and predict their behavior on the basis of these mental states).

\section{IMPROVISATIONAL ACTING}

Improvisational acting (improv) is a creative group performance where actors co-construct stories on stage in real time. Performances are constrained by sets of game rules and audience suggestions [13]. Audience suggestions (e.g. "do a scene about plumbers") often provide some semantic priming to help improvisers interpret events, formulate beliefs, and build and repair their mental models.

Improvisers interact through the process of proposing and responding to offers (i.e. proposals made by improvisers in a performance to add something to a scene) [13]. Based on an improviser's perception of other improvisers' actions, he builds his hypothesis of his portrayed character, other agents' characters and the activity they are involved in. While making or responding to offers, an improviser is able to model other actors' mental models in the scene, evaluate the outcome of actions, and update goals, which can be referred to as theory of mind [19].

Improv actors negotiate their shared mental model solely through presentations to the ongoing scene rather than with explicit communication about the model [20]. Each improviser starts to act and build their individual mental model based on how he or she observes the world that is 
unfolding on stage. However, improvisers often make presentations that can be perceived or interpreted many different ways [20]. Actors may misinterpret dialogue or misinterpret pantomimed motions. For example, if one actor holds their fists one on top of the other and moves them from side to side, another actor could interpret this as either raking or sweeping, among other possibilities. To handle the ambiguities of these presentations, improv actors' mental models must deal with inexact information.

Improv actors execute motions on stage that are ambiguous. A simple motion like waving a hand can be interpreted multiple ways, including waving to someone, erasing a board, or cleaning a window. These ambiguous presentations and the ease with which improvisers can misinterpret them can cause an individual improviser to develop a mental model that differs from the other improvisers' models. The state where improvisers' mental models differ from each other is called cognitive divergence $^{2}$. Through processes of cognitive convergence, improvisers repair their divergent mental models to reach a state of cognitive consensus, where everyone agrees on the shared mental model [13]. Cognitive consensus can be thought of as the state of "being on the same page."

Improvisers employ different repair strategies to deal with divergences as they occur [13]. Repair strategies are either other-oriented or self-oriented. Other-oriented repair strategies aim to affect someone else's mental model through presenting new concepts (presentation) or correcting divergences (clarification). Self-oriented repair strategies try to align one improviser's mental model with others' by learning what the other improvisers think is going on. An actor may communicate an idea about what is going on in the scene so that other actors may confirm it (verification). Alternatively, the actor may introduce new, vague information to the scene in order to observe how the other improvisers react, hoping that this will reveal some disambiguating information (blind offer). Repair strategies help the agents update their mental models and approach cognitive consensus.

\section{RELATED WORK}

Virtual improvisational theatre has been the focus of research in digital improv systems. The ComputerAnimated Improvisational Theater (CAIT) offered two types of improv agents: Improv Puppets and Improv Actors [21], [22]. Both types of agents could improvise with other agents in real-time under the guidance of human input for how they should act. These agents reason about each other's mental models and can change their own mental model to conform to another's based on observations.

\footnotetext{
${ }^{2} \mathrm{We}$ currently ignore models built between the actors and the audience, though divergences can exist between them as well.
}

However, CAIT cannot allow humans to improvise with these systems as equal co-creators of a narrative because these agents could not create narratives on their own as a result of the absence of the procedural knowledge required for this act. Instead, they worked from pre-authored narrative structures. In the Improv system [23], virtual animated avatars could be scripted to enact a scenario giving the appearance of believable improvisational performances. Neither of these systems, however, applied a deep understanding of how professional actors perform improvisation.

Work on computational improvisational agents has yielded agents that model the cognitive processes central to the human act of theatrical improvisation. One set of agents played the improv game Party Quirks, where a human party host tried to discover what characters party guest agents portrayed. The agents needed to develop a shared mental model with the human in order to reach cognitive consensus [20]. However, the agents in Party Quirks can only reason about explicit true and false beliefs; they could not reason about information that was partially true [39].

The work described in this paper differs from other work done on joint intentions and shared beliefs, such as STEAM [24], ARITMIS [25], Collagen [26], the STAPLE architecture [27], and work done by Kennedy and Trafton [28]. The common aspect between these architectures is the presence of an explicit representation of team goals, plans and joint commitments, and the use of shared plan theories. Despite the existence of some similarities between STAPLE and our architecture, the main difference appears in the ability of our agents to deal with implicit shared mental models where one agent does not have to explicitly declare its beliefs to other agents. On the other hand, the work done by Kennedy and Trafton on simulation of shared mental models relies on hypothetical declarative knowledge and productions to help the agent to reason about the environment. This can hardly be achieved in a domain like improv because of the absence of pre-authored states that can aid in rule production.

THESPIAN is another system developed by Si et. al [19] that allows the agents to behave based on models of emotions and social normative behaviors in addition to having explicit author-specified plot design goals, but for the agents to decide on an action they are offered bounded look-ahead policy. Since Thespian agents cannot act in the absence of explicit goals and an explicit agreement between the agents and each other, they cannot be used in improvised/co-creative narrative experience.

\section{COMPUTATIONAL SHARED MENTAL MODELS FOR IMPROV AGENTS}

The work presented in this paper employs fuzzy logic and situation calculus to provide a mechanism that allows AI agents to reason about uncertain beliefs with degrees of 
truth and variable confidence. The fuzzy logic representations we describe here for reasoning, decision making, and shared mental models are integral parts of the Co-creative Cognitive Architecture (CoCoA) (Figure1). The components of $\mathrm{CoCoA}$ form the knowledge that improv agents use to understand and contribute to an emergent narrative that they co-create with a human user.

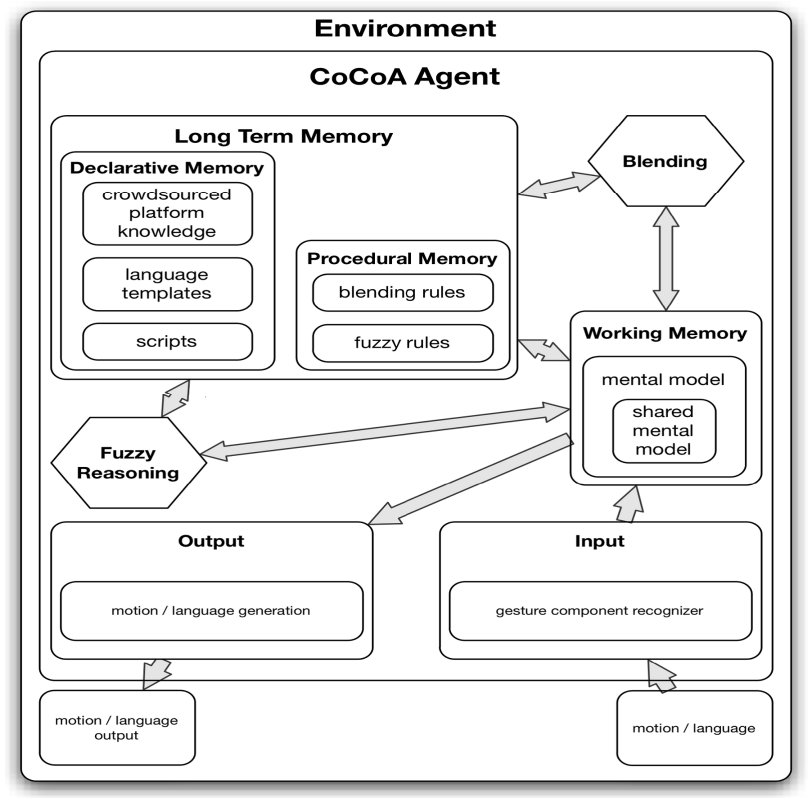

Figure 1. The CoCoA Architecture for Improv Agents

Figure 1 describes the main architecture of this work where the input layer contains a gesture component recognizer (a Microsoft Kinect connected to a specialized gesture code layer), which provides the means to capture a human interactor's posture and gesture. It is worth noting here that the language model has not been completed yet. The Kinect along with the code layer interpret the captured gestures and provides a list of possible actions that can be represented by that posture and gesture. This information is passed to the working memory, which uses fuzzy reasoning (the main focus of this paper) and long term memory knowledge to reason about this information. After the agent decides on his next action based on the knowledge retrieved from the input layer and the fuzzy reasoning module, the decision is passed to the motion/language generation in the output layer, which displays an animated AI agent performing that action as an output.

\section{Human Reasoning and Fuzzy Logic}

Human reasoning can be defined as the process of manipulating symbols in order to improve knowledge and make better decisions [22]. One of the strengths of how humans reason about the world is the non-Boolean manner followed when building mental models, which allow them to reason about vague and imprecise knowledge [14]. Mental models not only contain such knowledge but also information about how to use this knowledge. This nonBoolean description of categorical knowledge is informed by contemporary views in cognition and category theory, such as work by Lakoff [30] and Rosch [31]. One way to represent this knowledge is fuzzy logic, which reduces the design complexity of "humanistic" systems, which usually operate in a decision-making environment involving vagueness, imprecision and uncertainty.

In Boolean logic, any concept has only two possible values for its membership in any set: True and False. Fuzzy logic allows the notion of membership in a set to become a matter of degree [32]. In other words, an element $x$ has a degree of membership (DOM) that ranges from 0 to 1 to indicate how much it belongs in a fuzzy set $S$. A DOM of 0 means $x$ is definitely not a member of $S$ (false in Boolean logic). A DOM of 1 means $x$ definitely is a member of $S$ (true in Boolean logic). For example, a person of 2 meters high may have a DOM of 0.9 in the set of tall person and a DOM of 0.3 in the set of short person. Degree of association (DOA) is defined as the degree of membership between two elements in two knowledge categories. In other words, DOAs provide a bidirectional version of degree of membership that indicates how much two elements are associated with each other [23].

\section{Shared Mental Model for Improv Agents}

Shared mental models emerge from agents' individual mental models as a perceived shared construct of what everyone knows and thinks. SMMs among team members develop from working and training together on similar tasks [33-35] and from things like team experience and common organizational familiarity.

The aim of our work described in this paper is to design and implement a shared mental model representation for cocreative improvisational agents. The shared mental model is not a shared knowledge construct but rather a socially agreed upon one that resides as two separate models (one in each agent). Yet, cognitive convergence is achieved when the shared mental models coincide as shown in Figure 2.

The main challenge in designing improv agents that can construct shared meaning as part of a performance is to deal with the absence of explicit coordination between agents, either as part of the improvised interactions or behind the scenes. Rather, a shared mental model need be constructed during the narrative co-construction/performance.

In an improvised scene, the agents communicate with each other through actions. This form of give-and-take communication helps each agent to understand what the other agents' beliefs might be and consequently allow both mental models to converge to a single model (i.e. shared mental model). Our shared mental model incorporates common individual views of both agents' mental models, see Figure 2. Each individual mental model consists of three components: beliefs, commitments, and reasoning and 
decision-making modules. The beliefs component models the agent's beliefs about itself, other agents and the surrounding world. Those beliefs are associated with confidence factors that show how much the agent thinks his belief is correct. For example, BEL(agent, is (agent, sheriff), medium, 0.513). This rule states that agent $\mathrm{x}_{\mathrm{x}}$ has medium confidence in agent being a sheriff and this confidence is equal to 0.513 .

The commitments component encapsulates any obligations (commitments) the agent might have towards other agents. An example of the rules used in this component is COM (agent $_{\mathrm{y}}, \mathrm{p}$ ), which states that agent $\mathrm{y}_{\mathrm{y}}$ is committed to respond to last action agent $\mathrm{x}$ performed ( $\mathrm{p})$. Finally, the reasoning and decision-making component provides feedback that notifies the mental model about any inconsistencies that exist (explained later in this paper). For example, the agent might have a certain belief about his partner's character and a new belief about his partner's last action, if the module found that this action cannot be performed by that particular character, an inconsistency flag is raised and the agent has to make a decision on keeping only one of those beliefs based on his confidence in both. Moreover, the reasoning and decisionmaking component allows the agent to decide on its next move (action) based on his current mental model.

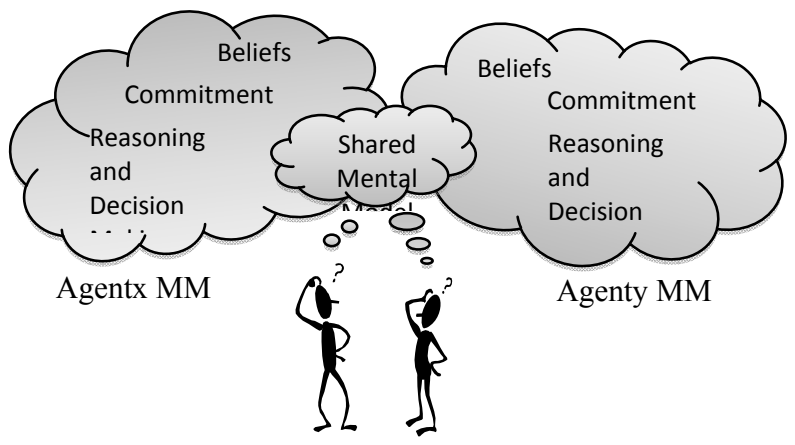

Figure 2. Representation of improv agents' mental models

To formalize the components of a shared mental model computationally, we utilize a hybrid model that incorporates confidence factors, iconicity, probability, and fuzzy logic along with situation calculus. The next sections touch on each of these techniques.

\section{Confidence Factor}

In order for an agent to act on a piece of knowledge, the knowledge must exist and the agent must be aware of its existence [36]. AI agents can capture the notion of awareness by unambiguously having an explicit fact that the agent knows specific knowledge. However, these facts will not account for changes in beliefs over time. This can be remedied through the introduction of confidence factors that dynamically change based on changes in the world. Confidence factors are fuzzy values (DOMs on a scale from 0 to 1) that allow an agent to compute the strength of its beliefs in a specific world frame, i.e. at any instant during the scene. For example, in an improvised act, at one instance one improviser can believe his partner is a sheriff with medium confidence and at another instance the same improviser can decrease his confidence in this belief based on their mutual interactions in the scene.

\section{Iconicity Function}

A main part of the knowledge that improvisers employ in a scene can be represented by degrees of association between different content and presentation aspects of a scene [37]. Content aspects are part of what improvisers call the platform, which includes the scene's location, the characters and their relationship to each other, and the joint activity the characters are participating in [8]. Presentation aspects include the motions improvisers make, the semantic actions these motions represent, and the dialogue improvisers speak. Agents use the DOAs between elements in this knowledge category to interpret presentations from other actors, extrapolate new information about the scene to add to their own mental model, and decide what information to present and how (see Figure 3).

Agents do not always present an element (e.g. a character) in a scene that has the highest DOA to the other elements in its mental model. Rather, an improviser often presents something that is iconic, something that conveys a significant amount of information about the agent's mental model [20]. An element is iconic if it is one of a few elements in a knowledge structure category with its particular DOA to a given element in another category. For example, in the context of an Old West story, only a few characters, like an outlaw, are highly associated with robbing a bank. Therefore, robbing a bank is an iconic activity for an outlaw and, if an actor starts a scene with a bank robbery, they are suggesting very highly that their character is an outlaw. On the other hand, many characters are highly associated with riding horses. Riding a horse is not an iconic activity for an outlaw; it does not provide a lot of information about which character the agent is trying to portray. When an agent makes an iconic presentation, another improviser is less likely to misinterpret the presentation, which reduces cognitive divergences and aids in repairing the shared mental model.

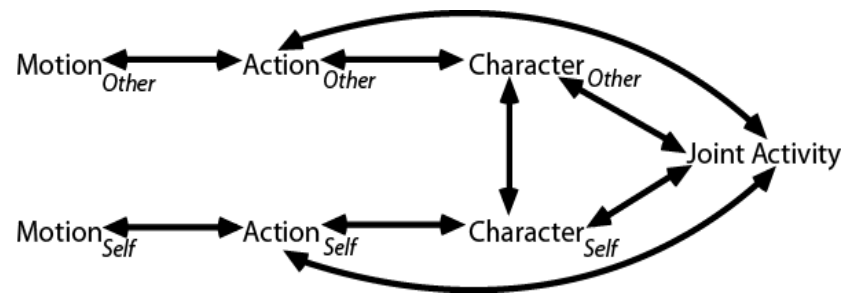

Figure 3. Degree of association (DOA) relationships between knowledge categories

Figure 3 shows the four categories of the knowledge base that exist in the agent's mental model: motions, actions, characters, and joint activities. Those categories are linked via DOAs in a way that allows an agent to infer new knowledge from existing ones. Based on the agent's 
confidence in his mental model, he decides to either perform an action or extrapolate to new knowledge categories first based on the s DOAs links. For example, if the agent has information about Action $_{\text {other }}$ (an action done by the other agent) it can extrapolate to Character ${ }_{\text {other }}$ (the other agent's portrayed character) and/or joint activity (the activity the agents are performing together).

Iconicity calculations are similar to those used in [11] for Party Quirks. A typical game of Party Quirks involves four players: one plays the role of party host, and all others play as party guests. Each party guest is assigned a "quirk" - some special trait for each guest that is public knowledge to everyone except the party host, including the audience. The host player then, within the context of hosting a party, aims to figure out what quirk each guest is portraying through their interactions. A guest typically leaves the scene when the host has successfully guessed their quirk. In Party Quirks, iconicity values are computed between two element categories only but these are updated to account for the different element categories of the knowledge structure. To calculate iconicity, we take a pair of elements from two different knowledge structure categories (element $e_{1} \in$ category $C_{1}$, element $e_{2} \in$ category $C_{2}$ ). The iconicity of this pair of elements depends on how far its DOA value is from the average DOA value for pairs of all elements of category $C_{1}$ to element $e_{2}$ (how easy it is to tell that $e_{1}$ is unusual) and how many other elements of $C_{l}$ have a similar DOA value (how many possible elements $e_{1}$ could be confused with), as shown in Equation 1.

Equation 1: Iconicity of $<e_{1} \in C_{1}, e_{2} \in C_{2}>=$

$1-\left[\left(1-\left|\operatorname{avgDOA}\left(<\forall e \in C_{1}, e_{2} \in C_{2}>\right)-\operatorname{DOA}<e_{1} \in C_{1}, e_{2} \in C_{2}>\right|\right.\right.$

) ] / ( (number of $<\forall e \in C_{1}, e_{2} \in C_{2}>$ pairs) - (number of $<\forall e \in C_{1}$, $e_{2} \in C_{2}>$ pairs within $1 \sigma$ of $\mathrm{DOA}<e_{1} \in C_{1}, e_{2} \in C_{2}>$ ))

In other words, the iconicity of a pair of two elements from different categories is [1- $(1-$ absolute value of (the average of all DOA values for elements of $C_{1}$ and element $e_{2}-\mathrm{DOA}$ between $e_{1}$ and $\left.e_{2}\right)$ ) / (the number of elements in $C_{1}$ - the number of elements of $C_{1}$ with a DOA to $e_{2}$ within one standard deviation of the DOA between $e_{1}$ and $e_{2}$ )]. Iconicity values are all normalized on a scale from 0 to 1 .

\section{Probability of Knowledge Selection}

An agent can use the iconicity of the elements in its mental model to determine the probability that a given element will be selected from its knowledge category given the current state of its mental model. In other words, despite the fact that it seems reasonable for an agent to present an element with high probabilistic value, this is not always the case because humans do not always do this. Humans can sometime choose to do something that seems reasonable in a specific situational rather than doing another action that seem more probable to be executed in that situational based on the way they perceive the situational (their mental model). On the other side, probabilities for choosing elements in the agent's mental model help it to estimate/measure its confidence that its mental model is correct.

The probability function for mental model elements is given by the following equation:

Equation 2: Probabilistic value of $e_{1} \in C_{1}$ given $e_{2} \in C_{2}=$ (Iconicity of $\left\langle e_{1} \in C_{1}, e_{2} \in C_{2}>/ \Sigma\right.$ Iconicity $<\forall e_{1} \in C_{1}, e_{2} \in C_{2}>$ ) That is, the probability that $e_{1}$ is the value for $C_{1}$ if $e_{2}$ is the value for $c_{2}$ is (the iconicity of $e_{1}$ in $\left\langle e_{1} \in C_{1}, e_{2} \in C_{2}>/\right.$ the sum of the iconicities for all elements in $C_{1}$ given $e_{2}$ ).

\section{Fuzzy Logic}

Fuzzy logic is a valuable tool for dealing with vague knowledge and the approximate nature of human reasoning [30]. It allows reasoning in the presence of linguistic variables that are vague and have no concise definition, such as temperature, probability and confidence using membership functions.

For a linguistic variable $\mathrm{X}$, membership functions on $\mathrm{X}$ represent fuzzy subsets of $\mathrm{X}$. The membership function, which represents a fuzzy set $A$, is usually denoted by $\mu_{\mathrm{A}}$. For an element $\mathrm{x}$ of $\mathrm{X}$, the value $\mu_{\mathrm{A}}(\mathrm{x})$ is called the membership degree of $\mathrm{x}$ in the fuzzy set $\mathrm{A}$. The membership degree $\mu_{\mathrm{A}}(\mathrm{x})$ quantifies the grade of membership of the element $x$ to the fuzzy set $\mathrm{A}$. The value 0 means that $\mathrm{x}$ is not a member of the fuzzy set; the value 1 means that $\mathrm{x}$ is fully a member of the fuzzy set. The values between 0 and 1 characterize fuzzy members, which belong to the fuzzy set only partially.

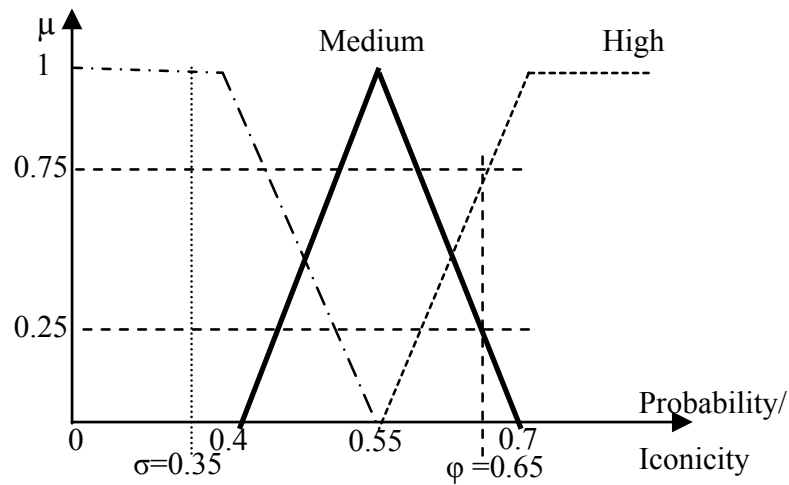

Figure 4. Diagrammatic representation of fuzzy probabilities and iconicity values using trapezoidal and triangular membership functions. The low, medium, and high fuzzy values are used to determine the agent's confidence based on the probability $\varphi$ and iconicity $\sigma$.

For the purpose of this work, we decided to treat iconicity, probability, and confidence factors as fuzzy variables with fuzzy sets low, medium, and high. The aim is to use iconicity and probability input values to compute a confidence value utilizing Triangular and trapezoidal membership functions.

Mapping iconicity (Equation 1) and probabilistic interpretations of likelihood informed by the iconicity function (Equation 2) to a corresponding confidence factor was done through the use of Trapezoidal and Triangular Membership Functions for our fuzzy values (low, medium, and high) (Figure 4). For the membership function of 
probability, the $\mathrm{x}$-axis represents the inputs of the probabilistic values $\varphi$ resulted from the iconicity function. The y-axis represents the degree of membership $\mu$ of element $\varphi$ in the fuzzy sets low, medium, and high, where each term $x$ in Probability has a $\mu_{\text {probability }}(\mathrm{x})$ characterized by a fuzzy set in a universe of discourse $\mathrm{U}=[0,1]$.

We designed fuzzy rules to evaluate the output confidence factors for iconicity and probability input values. Fuzzy rules can be expressed in "if... then..." form. The "if..." part is called the antecedent and the "then..." part is called the consequent. Both parts contain linguistic variables defined by fuzzy sets. A linguistic variable can be either a variable whose value is a fuzzy number or values defined in linguistic terms.

Choosing the linguistic values low, medium and high for the iconicity $(\mathrm{I})$, probability $(\mathrm{P})$, and confidence factors $(\mathrm{CF})$ variables, the following fuzzy inference rules were designed:

$R_{1}$ : IF I is high and $P$ is high THEN CF is high

$R_{2}$ : IF I is high and $P$ is medium THEN CF is medium

$R_{3}$ : IF I is high and $P$ is low THEN CF is medium

$R_{4}$ : IF I is medium and $P$ is high THEN CF is medium

$R_{5}$ : IF I is medium and $P$ is medium THEN CF is medium

$R_{6}$ : IF I is medium and $P$ is low THEN CF is low

$R_{7}$ : IF $I$ is low and $P$ is high THEN CF is medium

$R_{8}$ : IF $I$ is low and $P$ is medium THEN CF is medium

$R_{9}$ : IF I is low and P is low THEN CF is low

The designed rules are sufficient to serve the purposes of this work. Consider now an iconicity value of 0.65 and probability of 035 . According to the Trapezoidal and Triangular membership function, the iconicity of 0.65 has 0 membership in the low fuzzy set, 0.25 membership in the medium fuzzy set, and 0.75 membership in the high fuzzy set (see Figure 4). The 0.35 probability has 1 membership in the low fuzzy set, 0 membership in the medium fuzzy set, and 0 membership in the high fuzzy set. According to the above noted IF-THEN rules, using fuzzy AND then the fuzzy output can be obtained as follows:

\begin{tabular}{|l|c|c|c|}
\cline { 2 - 4 } \multicolumn{1}{c|}{} & \multicolumn{3}{c|}{ Membership Grade } \\
\cline { 2 - 4 } \multicolumn{1}{c|}{} & Low & Medium & High \\
\hline Rule 1: $\min (0.75,0)$ & & & 0 \\
\hline Rule 2: $\min (0.75,0)$ & & 0 & \\
\hline Rule 3: $\min (0.75,1)$ & & 0.75 & \\
\hline Rule 4: $\min (0.25,0)$ & & 0 & \\
\hline Rule 5: $\min (0.25,0)$ & & 0 & \\
\hline Rule 6: $\min (0.25,1)$ & 0.25 & & \\
\hline Rule 7: $\min (0,0)$ & & 0 & \\
\hline Rule 8: $\min (0,0)$ & & 0 & \\
\hline Rule 9: $\min (0,1)$ & 0 & & \\
\hline
\end{tabular}

Table 1. Membership grade of the inference rules

$\left[\mathrm{A}_{\mathrm{j}}\left(\mathrm{x}_{\mathrm{o}}\right) \wedge \mathrm{B}_{\mathrm{j}}\left(\mathrm{x}_{\mathrm{o}}\right)=\mathrm{m}_{\mathrm{j}}\right.$, where $\mathrm{m}_{\mathrm{i}}$ is the membership of the output of each rule. Then with $\mathrm{w}_{\mathrm{j}}$ denoting the crisp output of rule $\mathrm{j}$ as shown in (1), the Centroid Defuzzification
Method for the outputs shown in Table 1 applies as follows to produce the crisp output $\mathrm{CF}$ : $\mathrm{CF}=\Sigma_{\mathrm{i}=1} \mathrm{~m}_{\mathrm{i}} * \mathrm{~W}_{\mathrm{i}} / \sum_{\mathrm{i}=1} \mathrm{~m}_{\mathrm{i}}$ In this example, $\mathrm{CF}=[(0.75 * 0.55)+(0.25 * 0.4)]$ / $(0.75+0.25)=0.513$, where $0.4,0.55$ and 0.7 are the centers of gravity of the fuzzy outputs low, medium and high.

The agent can now use this confidence value to represent how confident it is in a specific belief in his mental model as follows: BEL (agent , is (agent $_{y}$, sheriff), medium, 0.513). In plain English, this predicate can be read as: agent ${ }_{x}$ believes that agent $\mathrm{y}_{\mathrm{y}}$ is a sheriff with medium confidence equal to 0.513 . Medium value is chosen here based on the ranges chosen for the data value sets low, medium and high.

\section{Updating Mental Model Beliefs}

The previous section illustrates how an agent can compute their confidence about a specific belief. It is worth noting that the agent's beliefs can change as a scene unfolds. Consequently, each agent should be able to update its beliefs and associated confidences based on what it infers from the introduced information. Hence, we designed more fuzzy rules that can help the agent to update his beliefs as the following example:

Fuzzy Rule One: IF agent $\mathrm{x}_{\mathrm{x}}$ has low confidence about agent $\mathrm{x}_{\mathrm{x}} \mathrm{s}$ motion $A N D$ has medium confidence about agent ${ }_{\mathrm{x}}$ as character $_{\mathrm{y}}$,

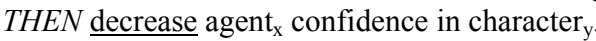

This rule is designed to decrease agent B's confidence about agent A's character if agent A did a motion that can be interpreted as related to two different characters $\mathrm{X}$ and Y. For example, if one agent holds their fists one on top of the other and moves them from side to side to perform a sweeping motion, an agent could relate this motion to both a waiter character and a janitor character. Other rules were designed to increase the agent confidence about his beliefs.

The agents' confidence about the knowledge in their mental models keeps changing based on the actions they take to establish a shared mental model and consequently reach cognitive consensus. Reaching cognitive consensus requires the agents to have the same beliefs as a subset of their individual beliefs. The next section defines how shared mental models make use of various strategies to address cognitive divergence whenever recognized and how this helps the agents to approach cognitive convergence.

\section{SITUATION CALCULUS AND REPAIR STRATEGIES}

SMMs are a hypothetical cognitive construct that extends the notion of individual mental models to a team context [17]. Based on an empirical study of the cognition employed by performers in improvisational theatre that focuses on how improvisers create a dramatic or comedic performance in real-time, it has been found that in an improvisational scene, improvisers employ different repair strategies to deal with divergences as they occur and that they rely on their shared mental model to decide on the suitable repair strategy to use [13]. Repair strategies are 
those strategies utilized by improv actors to achieve cognitive consensus when divergences arise. They help the agents update their mental models and approach cognitive consensus. The choice of a repair strategy depends on the status of the agent's mental model in a specific frame of the world. Due to the dynamic nature of the agent's shared mental model, situation calculus is appropriate for the representation of repair strategies because of its ability to capture change based on time along with reasoning about causes and effects of these changes.

Situation calculus is a logic formalism designed for representing and reasoning about dynamic domains [38]. Situation calculus is one way to represent changes in First Order Logic by adding a situational argument to each noneternal predicate. We used fluents such as BEL ( ) to indicate whether an agent has a specific belief about a relation or a proposition or not and function fluents to describe the state of the world, for example the CONF ( ) function returns how confident agent $\mathrm{x}$ is about certain knowledge. Now we have introduced situation calculus, we are going to explain how it is used to represent the repair strategies.

\section{Repair Strategies}

Presentation is another-oriented repair strategy (i.e. attempts to change other's model instead of their own). It is the foremost repair technique that demonstrates what an improviser believes to be true and introduces new information that relates to an individual's mental model. Clarification is another other-oriented repair technique used by improvisers to correct any misunderstandings or misinterpretations of information that has already been communicated. It is different from presentation in that it does not introduce any new concepts (unless those new concepts are meant to clarify old ones). For various types of clarification, see [13].

Verification is a self-oriented repair technique. Verification occurs when an improviser has an idea of what another improviser's mental model might be, and he often communicates his impression to his scene partner(s) in order to verify it. Verification is not always an exact formulation of what an improviser believes. It can also manifest as a statement that is related to a belief that an improviser wants to test for accuracy (i.e. wants to verify). This is one repair strategy that relies on dialogue in improv theatre, however as we do not deal yet with dialogues between agents, situation calculus rules focus only on actions as follows:

A blind offer (related to the canonical improv offer, which is when an improviser presents a new potential contribution to a scene) is the final self-oriented repair technique. Improvisers use this term to describe when they intentionally introduce new, vague, and poorly-defined information. The purpose of this action is for the improviser's scene partner(s) to take the information and expand upon it, using the blind offer as an opportunity to present or clarify their mental model(s). The designed situation calculus rules are as follows:

Each of the above strategies do also have action effects axiom that commits the agent to respond to the interactor's action and successor state axiom that defines how the agent beliefs should change after the execution of the action.

\section{Acknowledgment of Repair Strategies}

After an attempted repair, an acceptance state follows that identifies if a repair strategy has failed or succeeded. A repair attempt is said to have failed if the divergence continues or a new one takes its place. The other possibility is that the repair succeeds, which means is met with acceptance [13]. Acceptance does not necessarily equate to cognitive consensus, but only defines a state that follows a repair attempt. The ideal form of consensus is true cognitive consensus, which is when an improviser correctly accepts another improviser's mental model. True cognitive consensus can only be identified through explicit confirmation by all the improvisers originally involved in the divergence (a phenomenon we capture in our group interview data [13]). Cognitive consensus, however, can be partial, which means that it is not necessary for all mental models to be identical, but at least two mental models that used to be different have become the same (one divergence has been resolved).

\section{EVALUATION}

In order to evaluate SMM presented in this paper, we ran two studies. The first study includes a live interaction between a human interactor and our AI agent. The second one includes a series of tests to measure the success of the two AI agents in their attempts to reach consensus in their beliefs.

\section{Study I}

In this study, we allowed a human interactor to interact with our improv agent. The agent starts with a mental model that contains only different elements in each of the knowledge categories defined earlier in Figure 3 along with DOAs between one another. Figure 5 shows the portion of the big network in which sheriff and cowboy are elements in the character category. Hold gun and punch are elements in the action category and bar-fight is an element in the joint activity category. 


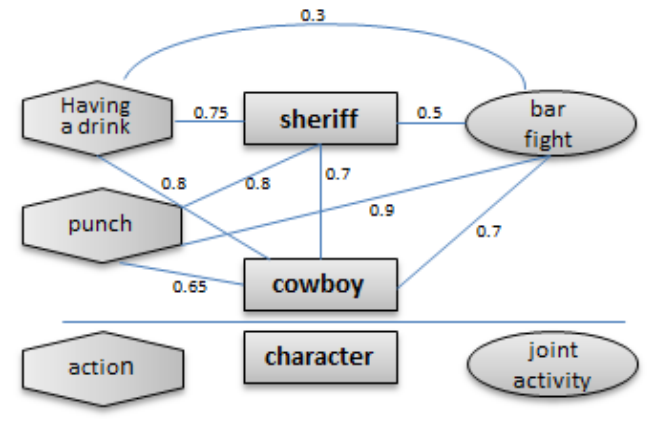

Figure 5. DOMs between, actions, characters, and joint_activities. Sheriff has very strong $(0.95)$ degree of association to hold gun action and medium (0.7) degree of association to the cowboy character. Both sheriff and cowboy have strong and medium DOAs

The player starts to interact with the improv environment using Microsoft Kinect. Now the human interactor is considered as agent $\mathrm{y}_{\mathrm{y}}$ and the improv agent is agent $\mathrm{x}_{\mathrm{x}}$. The human interactor starts by holding her hand cupped near her mouth as if she is having a drink. The agent recognizes the motion and extrapolates to action other which is the "having a drink" action. At this point, the agent can extrapolate to either character other or joint activity as shown in Figure3. As seen in Figure 5 'having a drink' action has DOAs with the 'cowboy' and 'sheriff' characters. Since there is no prior information about the scene, the agent just picks the character with the highest association that is cowboy and the following belief is added to the agent's mental model: BEL (agent $x_{x}$, is (agent $t_{y}$, cowboy), medium, 0.513). This beleief shows that the the improv agent has a high confidence that the human intercator is a cowboy. On the other hand, another belief is added in the long term memory that the human interactor might be a sheriff with medium confidence equal to 0.75 .

Based on the current mental model, the agent chooses to extrapolate to his character (character self) and decides on being an "outlaw". The agent then chooses an action to execute, which was "punch" action. The human interactor "punches" back. Now the agent has to update his confidence about the interactor's character based on the latest action using the rules in Section IV .C. The "punch" action has a medium DOA with the "cowboy" character so the final confidence is updated based on the following fuzzy rule.

Fuzzy Rule: If agent $_{\mathrm{x}}$ has medium confidence about agent 's $_{\mathrm{y}}$ motion $A N D$ has high confidence about agenty character, Then decrease agent $\mathrm{x}_{\mathrm{x}}$ confidence in agent $\mathrm{y}_{\mathrm{y}}$ character.

Using this knowledge the agent decides to extrabolate to the joint activity which results in adding the following belief

BEL (agent, joint_activity(agent ${ }_{y}$, bar-fight), medium, 0.513).

The agent chooses to do the "shoot-gun" action. Although the human interactor realized it is a bar-fight, she decided to change the context and chose to make the "sweep a table" move as shown in Figure 6. The agent interprets the move as "sweep table" action but according to his model this action is not likely associated with the cowboy character so he recognizes this as a divergence.

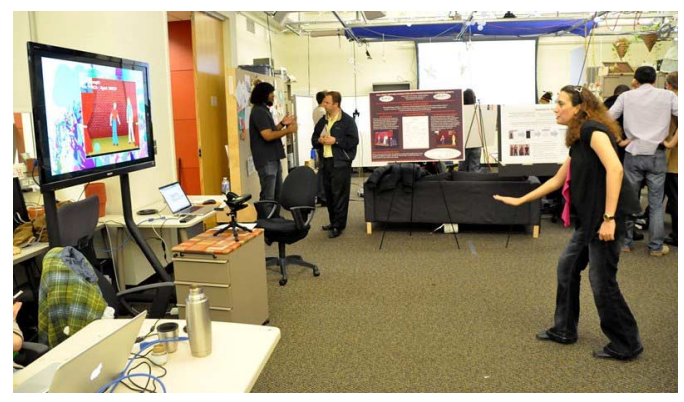

Figure 6. The human interactor is doing the 'sweep a table' action

To address this divergence, the agent decides on the appropriate repair rule to use. Since the agent has credible evidence (medium to high confidence) that he and the human interactor were doing the "bar fight" joint activity, the agent decides to do a clarification repair strategy- that is to amend his partner's mental model. Accordingly, based on the clarification definition, the agent decides to execute an action that is highly associated with his character, so he did the "raise hands up" action by raising his two hands up in a surrender position. It is worth mentioning that in the absence of the repair strategies the agent will keep just responding to the interactor's actions and would not take the initiative to try to change the interactor's mental model.

The human interactor realizes that the agent is still taking the role of an "outlaw" so she acknowledges the repair and chooses to go along with the agent so she decides to be the "sheriff" and moves forward as if she approaches the agent. The agent increases his confidence in the interactor's character and the joint activity; sheriff and bar-fight. The human interactor continues interacting with the agent a couple more times before the agent confidence passes a specified threshold of $90 \%$ so the agent announced that he thinks they reach consensus and that he thinks he is an "outlaw", the human interactor is a "sheriff" and they were involved in a "bar-fight".

This study provides some interesting insights about the capability of the shared mental model and how it can serve in producing interesting human computer interactions. Despite the fact that there were some mis-interpretations from both the interactor and the improv agent sides, it was still interesting to see the back-and-forth negotiation between the human interactor and the improv agent. It seems that the fuzzy rules handled the ambiguity and uncertainty embedded in the interactions in a satisfactory way as the human interactor ended up with an enjoyable improv experience. 


\section{Study II}

In the second study, we ran the two agents without allowing them to reason about the world in any way (choose their actions in a completely random manner). Each agent would guess at his own character, the other agent's character, and their shared joint activity entirely randomly. In these circumstances, the agents would be expected to reach cognitive consensus (complete agreement on both characters and the joint activity in the scene) on a given line only $0.07 \%$ of the time, due to the improbability of guessing correctly from our large pool of permutations. After running twenty scenes, each starting with a "blank slate" (no beliefs in either agent's mental model) and each running for one-hundred lines, the agents never once found total consensus. We would only expect total consensus to be found approximately once in over 1500 lines, so this is not a surprising outcome.

In contrast to this control run, we turned the SMMs on and allowed the agents to act out a series of twenty scenes (each continuing for one-hundred lines) in which each agent would attempt to infer the other agent's character, his own character, and their mutual joint-activity based on the actions performed in the scene. Each would then choose actions to perform and present to the other agent based on the actions' DOAs to his current beliefs about his own character and the joint activity. Utilizing this reasoning, the agents found total consensus at some point in 9 out of the 20 runs. Additionally, they reached near consensus (agreement on all but one element in a scene) in seventeen out of twenty runs, and were "one away" from total consensus (or better) in $10.0 \%$ of the total lines performed, which is over 4.5 times more often than in the random trials.

These numbers show that, even in the absence of explicit information-sharing between the agents, the reasoning used to populate our shared mental models significantly improved the agents' ability to reach common beliefs about a scene. By inferring the beliefs of other actors based on the visible actions performed, each agent can negotiate and reassess his beliefs strategically based on its unique set of assumptions about character-action-activity relationships.

\section{CONCLUSION}

Mental models underlie everything that happens in a cocreated story. Since improv involves the perpetual invention and contribution of new information to a scene, there is a constant potential for errors regarding that information. Therefore, understanding how to create shared mental models and navigate those errors is especially important. The process of cognitive convergence allows improvisers to deal with their mistakes and reach an understanding, using shared mental models as the foundation for their construction of a collaborative scene.
This paper presents a shared mental model for improvisational narrative agents who rely on their beliefs and desires to achieve cognitive consensus in a coconstructed scene through shared mental models, which in turn provides a model for how humans and agents can cocreate a scene together through similar implicit means. Fuzzy logic and situation calculus were able to successfully handle the continuous change in the agent's mental model. Turning the SMM on and off proofs the validity of using confidence factors to guide the decision making and provides us with evidence on the validity of the model as it enhances the performance of the agents and allows them to reach cognitive convergence 4.5 times more often than in the random trials when the SMM was turned off.

The model presented in this paper allows the elicitation of implicit objectives that guide the agents to achieve cognitive consensus and recognize when it is achieved. This model provides the flexibility for improv agents to be employed in games environments where they can reason about fuzzy uncertain knowledge and interact with each other and with human users.

Although the generated repair rules were designed for the improvisation domain, they pretty much capture how the negotiation process occurs in other domains too. We always present new things to others (Presentation), ask for clarification when we are confused about something (Verification), or make attempts to clarify things to others (Clarification). Therefore, it seems that these rules are applicable in those domains that share common characteristics with the improvisation domain, such as argumentation (using natural language) or an AI-based dancing game (using gestures).

In its current state, our approach does not capture all of the complexities of a full theory of mind because assessments are only based on DOA. For example, agents with the kind of SMM described here cannot reason about privileged knowledge (e.g. knowledge that only one agent knows because the other agent was not present to hear it), which a full theory of mind can account for. Improv agents would need to reason about all aspects of theory of mind to be able to create all the same kinds of narratives a human can. However, the shared mental models proposed in this paper are enough for improv agents to begin improvising a scene while considering each other's beliefs. Most importantly, we would argue that this model serves as an important step in preserving high user agency and improvisational narrative generation simultaneously, particularly within domains that rely on natural interfaces as a way to introduce ambiguity in the communication between user and computer.

\section{ACKNOWLEDGMENTS}

Rania Hodhod and Brian Magerko gratefully acknowledge the grant from NSF (\#IIS 0757567). 


\section{REFERENCES}

1. D. L. Roberts and C. L. Isbell, "A survey and qualitative analysis of recent advances in drama management," Int. Trans. Systems Science and Applications, Special Issue on Agent Based Systems for Human Learning, vol. 4, no. 2, 2008.

2. M. Cavazza, F. Charles, and S. J. Mead, "Interacting with virtual characters in interactive storytelling," in Proc. $1^{\text {st }}$ Int. Joint Conf. Autonomous agents and multiagent systems: part 1, 2002, pp. 318-325.

3. R. M. Young, M. O. Riedl, M. Branly, A. Jhala, R. J. Martin, and C. J. Saretto, "An architecture for integrating plan-based behavior generation with interactive game environments," Game Development J., vol. 1, no. 1, pp. 51-70, 2004.

4. M. O. Riedl and R. M. Young, "Narrative planning: balancing plot and character," Artificial Intelligence Research J., vol. 39, no. 1, pp. 217-268, 2010.

5. B. Magerko, "Player modeling in the interactive drama architecture," Ph.D Dissertation, Dept. CS and Eng., Michigan Univ., Michigan, USA, 2006.

6. V. Bayon, J. R. Wilson, D. Stanton, and A. Boltman, "Mixed reality storytelling environments," Virtual Reality J., vol. 7, no. 1, pp. 54-63, 2003.

7. R. Aylett, M. Vala, P. Sequeira, and A. Paiva, "Fearnot!-an emergent narrative approach to virtual dramas for anti-bullying education," in Proc. Int. Conf. Virtual Storytelling. Using Virtual Reality Technologies for Storytelling, 2007, pp. 202-205.

8. R. K. Sawyer, Improvised dialogues: Emergence and creativity in conversation, $1 \mathrm{st}$ ed. Westport, CT: Ablex Publishing Corporation, 2003.

9. A. Brisson, B. Magerko, and A. Paiva, "Tilt Riders: Improvisational Agents Who Know What the Scene is About," in Intelligent Virtual Agents $10^{\text {th }}$ Int. Conf., IVA 2011, Reykjavik, Iceland, September 15-17, 2011. Proceedings, Reykjavik, Iceland, 2011.

10. R. de Moraes and A. Forrest, "Whose Line - Party Quirks," Whose Line is it Anyway?, ABC, Hollywood, CA, 04-Oct-2001.

11. Whose Line Is It Anyway? (UK), TV.com. Retrieved September 19, 2012 from http://www.tv.com/shows/whose-line-is-it-anyway-uk/

12. R. K. Sawyer, "Improvisational Cultures: Collaborative Emergence and Creativity in Improvisation," Mind, Culture, \& Activity, vol. 7, no. 3, pp. 180-185, Aug. 2000.

13. D. Fuller and B. Magerko, "Shared mental models in improvisational performance," in Proc. the Intelligent Narrative Technologies III Workshop, Monterey, CA, 2010, pp. 1-6.
14. M. J. Davidson, L. Dove, and J. Weltz. (1999, November). Mental models and usability. Depaul University, Cognative Psychology 404 [Online]. Available: http://www.lauradove.info/reports/mental\%20models. htm

15. R. Fagin and J. Y. Halpern, "Belief, awareness, and limited reasoning," Artificial Intelligence, vol.34, pp. 39-76, 1988.

16. I. van de Kieft, C. M. Jonker, M. B. van Riemsdijk, "Improving User and Decision Support System Teamwork: An Approach Based on Shared Mental Models," Workshop Explanation-aware ComputingIJCAI, Barcelona, Spain, 2011, pp. 61-70.

17. J. Yen, X. Fan, S. Sun, T. Hanratty, and J. Dumer, "Agents with shared mental models for enhancing team decision makings," Decision Support System J., vol. 41, pp. 634-653, 2006.

18. S. SPAULDING, "Embodied cognition and mindreading," Mind \& Language, vol. 25, no. 1, pp. 119-140, 2010.

19. M. Si, S. C. Marsella, and D. V. Pynadath, "THESPIAN: An Architecture for Interactive Pedagogical Drama," in Proc. 2005 Conf. Artificial Intelligence in Education: Supporting Learning through Intelligent and Socially Informed Technology, Amsterdam, The Netherlands, The Netherlands, 2005, pp. 595-602.

20. B. Magerko, P. Dohogne, and D. Fuller, "Shared Mental Models in Improvisational Digital Characters," in Proc. 2nd Int. Conf. Computational Creativity, Mexico City, Mexico, 2011, pp. 33-35.

21. B. Hayes-Roth, E. Sincoff, L. Brownston, R. Huard, and B. Lent, "Directed Improvisation," in Technical Report KSL-94-61, Palo Alto, CA, 1994.

22. B. Hayes-Roth and R. Van Gent, "Story-Making with Improvisational Puppets and Actors," in Technical Report KSL-96-09, Palo Alto, CA, 1996.

23. K. Perlin and A. Goldberg, "Improv: A System for Scripting Interactive Actors in Virtual Worlds," in Proc. 23rd Annu. Conf. Computer Graphics and Interactive Techniques, New York, 1996, pp. 205216.

24. M. Tambe, "Towards flexible teamwork," in Artificial Intelligence Research J. (JAIR), vol. 7, pp. 83-124, 1997.

25. M. D. Sadek, P. Bretier, and F. Panaget, "ARTIMIS: Natural dialogue meets rational agency," in Int. Joint Conf. Artificial Intelligence, 1997, vol. 15, pp. 1030 1035. 
26. C. Rich and C. L. Sidner, "COLLAGEN: A collaboration manager for software interface agents," User Modeling and User-Adapted Interaction, vol. 8, no. 3, pp. 315-350, 1998.

27. R. A. Subramanian, S. Kumar, and P. Cohen, "Integrating joint intention theory, belief reasoning, and communicative action for generating teamoriented dialogue," in Proc. THE NATIONAL Conf. ARTIFICIAL INTELLIGENCE, 2006, vol. 21, p. 1501.

28. W. G. Kennedy and J. G. Trafton, "Using simulations to model shared mental models," DTIC Document, 2007.

29. R.A. Subramanian, S. Kumar, and P.R. Cohen, "Confirming Changes in Beliefs and Intentions," in Proc. AAAI Spring Symposium: Intentions in Intelligent Systems, 2007, pp. 46-47.

30. G. Lakoff, Cognitive models and prototype theory. In U. Neisser (Ed.), Concepts and Conceptual Development: Ecological and Intellectual Factors in Categorization, Cambridge: Cambridge Univ. Press, 1987, pp. 63-100.

31. E. Rosch, Principles of Categorization. In E. Rosch and B. Lloyd (Eds.), Cognition and Categorization, Hillsdale, NJ: Lawrence Erlbaum, 1999.

32. J. Yen and R. Langari, Fuzzy Logic: Intelligence, Control and Information. $1^{\text {st }}$ ed., New York: Prentice Hall, 1998.

33. J. R. Rentsch and R. J. Hall, Members of great teams think alike: A model of team effectiveness and schema similarity among team members. In M. M. Beyerlein \& D. A. Johnson (Eds.), Advances in interdisciplinary studies of work teams: Theories of self-managing work teams, Greenwich, CT: JAI Press, 1994.
34. J. E. Mathieu, T. S. Heffner, G. F. Goodwin, E. Salas, and J. A. Cannon-Bowers, "The influence of shared mental models on team process and performance.," Applied Psychology J., vol. 85, no. 2, pp. 273-283, 2000.

35. L. L. Levesque, J. M. Wilson, and D. R. Wholey, "Cognitive divergence and shared mental models in software development project teams," Organizational Behavior J., vol. 22, no. 2, pp. 135-144, 2001.

36. L. O'Brien, Self-Knowing Agents. Oxford University Press, 2007.

37. B. O’Neill, A. Piplica, D. Fuller, and B. Magerko, “A Knowledge-Based Framework for the Collaborative Improvisation of Scene Introductions," in Proc. $4^{\text {th }}$ Int. Conf. Interactive Digital Storytelling, Vancouver, Canada, 2011.

38. Situation Calculus - Google Search. Retrieved July 2, 2012 from https://www.google.com/search?q=situational+calculu $\mathrm{s} \& \mathrm{ie}=\mathrm{utf}-8 \&$ oe $=$ utf-8\&aq $=$ t\&rls=org.mozilla:enUS:official\&client=firefox-a

39. B. Magerko, C. DeLeon, and P. Dohogne, "Digital improvisational theatre: party quirks," in Proc. $10^{\text {th }}$ Int. Conf. Intelligent Virtual Agents, 2011, pp. 42-47.

40. K. E. Weick and K. H. Roberts, "Collective mind in organizations: Heedful interrelating on flight decks," Administrative science quarterly, pp. 357-381, 1993.

41. S. Nicolle. Mental models theory and relevance theory.Pragmatics \& Cognition 11:2 (2003 ), $345-$ 378. 